\title{
Cerebrovascular pulsatility in patients with sleep-disordered breathing
}

\author{
Alberto R. Ramos, \\ Department of Neurology, Miller School of Medicine, University of Miami, 1120 NW 14th Street, \\ Suite 1350, Miami, FL 33136, USA \\ Digna Cabral, \\ Department of Neurology, Miller School of Medicine, University of Miami, 1120 NW 14th Street, \\ Suite 1350, Miami, FL 33136, USA \\ David J. Lee, \\ Department of Epidemiology and Public Health, University of Miami, Miami, FL, USA \\ Ralph L. Sacco, and \\ Department of Neurology, Miller School of Medicine, University of Miami, 1120 NW 14th Street, \\ Suite 1350, Miami, FL 33136, USA. Department of Epidemiology and Public Health, University of \\ Miami, Miami, FL, USA. Department of Human Genetics, University of Miami, Miami, FL, USA

\section{Tatjana Rundek} \\ Department of Neurology, Miller School of Medicine, University of Miami, 1120 NW 14th Street, \\ Suite 1350, Miami, FL 33136, USA. Department of Epidemiology and Public Health, University of \\ Miami, Miami, FL, USA
}

Alberto R. Ramos: aramos@med.miami.edu

\section{Abstract}

Purpose-The aim of our study is to determine the association between the pulsatility index (PI), a surrogate of cerebral small vessel disease and sleep-disordered breathing (SDB).

Methods-We conducted a transcranial Doppler ultrasound (TCD) study of 19 consecutive patients free of stroke and cardiovascular disease, referred for the evaluation of SDB. TCD was performed by a certified technologist. Subsequent polysomnography was performed according to the practice parameters of the American Academy of Sleep Medicine. We evaluated the association between the apnea-hypopnea index (AHI), the oxygen nadir, the blood flow velocities, and the Gosling PI, for the middle cerebral artery. We performed Spearman's rank correlation and nonparametric regression to evaluate the relationship between AHI, oxygen levels, and the PI.

Results-Median age was 48 years (range 37-83), with $52 \%$ male sex ( $n=10)$, and median BMI of 29.9 (range 25-40.4). The median AHI was 16.4 (0.2-69). The median PI was 0.97 (0.72-1.89) $\mathrm{cm} / \mathrm{s}$. The PI correlated with the AHI ( $\mathrm{rho}=0.44 ; p=0.004)$ and with age (rho $=0.57 ; p=0.001$ ). Nonparametric regression adjusting for age showed a positive association between the AHI and the PI (standardized estimate $=0.88 ; p=0.002$ ). There was no relation between the oxygen nadir and the PI.

Conclusion-We observed increased PI in patients with SDB during wakefulness. The PI could potentially be an estimate of cerebral small vessel disease in patients with SDB and hence allow evaluating cerebral hemodynamics during wakefulness with a clinically relevant device. 


\section{Keywords}

Transcranial Doppler; Sleep-disordered breathing; Pulsatility index; Stroke; Microvascular disease

\section{Introduction}

Sleep-disordered breathing (SDB) is an independent risk factor for ischemic stroke [1], but the pathological determinants of this relation are not well defined. Cerebral small vessel disease is increasingly considered an important mechanism in the pathophysiology between SDB and stroke. A higher percentage of silent ischemic brain lesions by magnetic resonance imaging have been observed in patients with moderate to severe SDB [2].

Transcranial Doppler ultrasound (TCD) is a safe, noninvasive technique widely used in clinical practice, which enables measurement of cerebral arterial blood flow velocities in the intracranial circulation [3]. The arterial pulsatility index (PI), calculated by TCD, is considered a measure of microangiopathic changes of cerebral blood vessels and indirectly reflects microvascular damage [3]. The aim of our study is to determine the association between PI, the apnea-hypopnea index, and the oxygen nadir in patients referred to a sleep disorders center.

\section{Methods \\ Population}

Nineteen consecutive patients referred for SDB were recruited from the sleep disorders clinic during their initial visit between April and October of 2011. Subjects were excluded if they had a history of stroke, carotid artery disease, cardiac disease, blood dyscrasia, coagulation disorder, and pulmonary disorder; or if they were previously treated with CPAP, surgery, or an oral appliance for SDB. Subjects using stable doses of hypertensive and oral hypoglycemic medications were not excluded from the study. The study was approved by the University of Miami Miller School of Medicine Institutional Review Board, and informed consent was obtained from all participants.

\section{Procedure}

All subjects completed a structured clinical interview, a neurological examination, TCD, and baseline PSG. Demographic characteristics, medication use, lifestyle, and sleep assessments were obtained through questionnaires, physical examination, and review of medical records. The heart rate and the systolic and diastolic blood pressures were obtained during daytime TCD monitoring by using a mercury sphygmomanometer.

\section{Nocturnal polysomnography}

Subsequent polysomnography (PSG) within 1 week of the clinical assessment and TCD was performed using a standard montage, including electroencephalographic, electromyographic (EMG), and electrooculographic [4] monitoring for an in-lab overnight video-PSG. Measures of airflow were taken to detect SDB. Sleep stages, arousals, and sleep-related events, apnea-hypopnea index (AHI), and nadir of oxygen desaturations were recorded according to the established practice parameters of the American Academy of Sleep Medicine (AASM) [5]. The sleep study was performed by certified technician in an AASMaccredited sleep laboratory. 


\section{Transcranial Doppler sonography}

The TCD was performed by a certified technologist (DC) using a portable microprocessorcontrolled TCD system on a laptop (EMS-9U, Delica) according to the guidelines of the American Institute of Ultrasound in Medicine [6]. A low-frequency (2 MHz) pulse-waved ultrasonic signal is transmitted from the skin surface across the cranial vault to the intracerebral vessels and receives the echoes along the same path. The TCD probe is positioned over "acoustic windows" that are specific regions of skull where the cranial bone is thin, allowing examination of middle cerebral artery (MCA) and the terminal portion of the internal carotid artery. The ultrasound beam emitted from the TCD probe reflects from the erythrocytes traveling within blood flow of the insonated artery. The transducer receives the signal and converts it into an electric signal. The following TCD variables are then analyzed: (1) peak systolic velocity (PSV), (2) end-diastolic velocity (EDV), (3) mean flow velocities (MFV), and, (4) PI. The PI is calculated by the Gosling equation, $\mathrm{PI}=(\mathrm{PSV}$ $-\mathrm{EDV}) / \mathrm{MFV}[3]$.

\section{Data analysis}

All analyses were carried out using SAS software version 9.2 (SAS Institute Inc, Cary, NC). Results are presented as median (range or interquartile range). The chi-square test was used to compare proportions, and Wilcoxon rank sum test was used for continuous nonparametric data. The Spearman's rank correlation was used to evaluate the relation between the AHI and oxygen nadir with demographic factors (age, sex, and BMI), blood pressure parameters (systolic blood pressure (SBP), diastolic blood pressure (DBP), and heart rate), and TCD measures (PI, MFV, PSV, and EDV). Nonparametric regression (Proc GAM) was used to analyze the association between the AHI and PI adjusting for age [7]. Further analysis was done by comparing the demographic, blood pressure, and TCD variables divided in two groups separated by the median AHI. Statistical significance was assumed for two-tailed $p$ values $<0.05$.

\section{Results}

The Table 1 presents the characteristics of the sample. The median age was 48 years, with $52 \%$ male sex $(n=10)$. The BMI was in the overweight-obesity range. Nine participants had hypertension and two had diabetes. The severity of SDB ranged from primary snoring to severe OSA based on the AHI. The PI correlated with the AHI (rho=0.44, $p=0.004)$ and with age (rho=0.57, $p=0.001$ ), while the oxygen nadir, sex, BMI, SBP, DBP, heart rate, and the Epworth sleepiness scale did not (data not shown). The AHI also correlated with age (rho $=0.44, p=0.05$ ), the oxygen nadir ( $r h o=-0.50, p=0.02$ ), and TCD end-diastolic velocity (rho $=-0.59, p=0.007$ ). The AHI did not correlate with sex, BMI, Epworth sleepiness scale, and the TCD measures of MFV and PSV. The group above the median AHI was significantly older, with lower end-diastolic velocity and increased PI (Fig. 1). There was no difference in sex, BMI, blood pressure parameters, and oxygen desaturation between the groups. Nonparametric regression adjusting for age showed an association between the AHI and the PI (standardized estimate $=0.88, p=0.002$ ).

\section{Discussion}

In our study, increased PI during wakefulness correlated with worse AHI. An increase in PI could mean a decrease in cerebrovascular compliance and cerebral small vessel disease $[8$, 9]. Similar results have been described in diabetes [10], Alzheimer's disease, and vascular dementia [8], but reports in patients with SDB are limited. In addition, increased PI is associated with white matter changes by magnetic resonance imaging (MRI), a marker of cerebral small vessel disease that predicts stroke, dementia, and disability [11]. 
We observed a median PI of 0.97 for the overall sample and 1.01 for the group above the median AHI. Our results are comparable to studies of microvascular complications in diabetes, where a mean PI of 0.87 was observed in the MCA of participants with complicated diabetes [10] and a mean PI of 1.05 in patients with diabetes and lacunar (small vessel) infarcts by MRI [12]. Several factors may influence the results of our study. Age and PI were correlated in our study; additionally, there was a positive correlation between age and AHI, with the group above the median AHI being significantly older. There is an increased prevalence and severity of SDB with age [13] and decreased cerebrovascular compliance associated with aging [8]. Furthermore, hypertension and diabetes can cause cerebral small vessel disease [14], but these factors did not correlate with the PI in our study. We observed an increased frequency of hypertension and a lower median score on the Epworth sleepiness scale in the group above the median AHI that did not reach statistical significance. In our clinical sample, the risk for SDB may be explained by snoring symptoms, obesity, and hypertension and less by the severity of the Epworth sleepiness scale. Our data are in agreement to findings of weak or no relationship between SDB and sleepiness score [15], but larger-sized clinical samples have correlated the degree of SDB severity to worse scores in the Epworth sleepiness scale [16]. Our findings could be partly explained by our small sample size and potential referral bias. SDB is closely related to hypertension and diabetes and may cause and/or exacerbate these vascular risk factors. Long-term exposure to hypertension and diabetes may have influenced the results of our study $[17,18]$. However, when controlling for age as the main confounder in our study, the AHI was still associated with increase PI. Our findings suggest that SDB could be an independent predictor of increased PI, but future studies on the link between SDB and subclinical measures of cerebrovascular disease are needed.

In our study, lower end-diastolic velocities during wakefulness were associated with worse AHI. This is in accordance with a study describing cerebral blood flow velocities in direct relation to apneic events. Abrupt increases in the PI have been described at the end of apneas were the MFV is the lowest $[9,19]$. The increased PI is primarily caused by a marked reduction in diastolic cerebral blood flow velocity. These changes follow the initial increase in systemic BP and cerebral blood flow velocities observed during the apneas, followed by a dip in end-diastolic and MFV $[9,20]$.

In our study, we did not detect an association between the oxygen levels and PI. Hypoxemia and hypercapnea are associated to impaired cerebrovascular reactivity to $\mathrm{CO}_{2}$ vasodilatory response [21]. The PI as a measure of cerebrovascular resistance may not directly quantify the effects of hypoxemia in the cerebral vasculature.

The mechanism, by which cerebral hemodynamic changes (PI) persist during wake, could be related to impairment of the endothelial and myogenic response to cerebral blood pressure fluctuations during the apneas [21]. These could contribute to atherosclerotic changes disrupting the arterial wall integrity, causing endothelial dysfunction and leading to cerebral small vessel disease [22].

Several limitations should be noted. First, there might be possible bias related to patient selection and the small sample size. The cross-sectional nature of our study does not allow assessing for causality and length of exposure to SDB. Even though we excluded participants with history of carotid disease, we did not asses for extracranial stenosis with carotid ultrasound. Several studies have shown an association between snoring, SDB, and carotid disease, but these findings have not been replicated in population-based studies [14]. Also, we do not think that this was a major source of bias, as our patients were relatively young without history of cardiac disease or stroke and few vascular risk factors. Finally, we did not apply positive airway pressure while recording the TCD. 
In conclusion, patients with SDB had increased PI during wakefulness. The PI could potentially be an estimate of cerebral small vessel disease in patients with SDB and hence allow evaluating cerebral hemodynamics during wakefulness with a clinically relevant device.

\section{References}

1. Redline S, Yenokyan G, Gottlieb DJ, Shahar E, O’Connor GT, Resnick HE, Diener-West M, Sanders MH, Wolf PA, Geraghty EM, Ali T, Lebowitz M, Punjabi NM. Obstructive sleep apneahypopnea and incident stroke: the sleep heart health study. Am J Respir Crit Care Med. 2010; 182:269-277. [PubMed: 20339144]

2. Minoguchi K, Yokoe T, Tazaki T, Minoguchi H, Oda N, Tanaka A, Yamamoto M, Ohta S, O'Donnell CP, Adachi M. Silent brain infarction and platelet activation in obstructive sleep apnea. Am J Respir Crit Care Med. 2007; 175:612-617. [PubMed: 17341649]

3. Gosling RG, King DH. Arterial assessment by doppler-shift ultrasound. Proc R Soc Med. 1974; 67:447-449. [PubMed: 4850636]

4. Somers CJ, Millar BC, Xu J, Moore DP, Moran AM, Maloney C, Keogh B, Murphy PG, Moore JE. Haemophilus segnis: a rare cause of endocarditis. Clin Microbiol Infect. 2003; 9:1048-1050. [PubMed: 14616751]

5. Epstein LJ, Kristo D, Strollo PJ Jr, Friedman N, Malhotra A, Patil SP, Ramar K, Rogers R, Schwab RJ, Weaver EM, Weinstein MD. Clinical guideline for the evaluation, management and long-term care of obstructive sleep apnea in adults. J Clin Sleep Med. 2009; 5:263-276. [PubMed: 19960649]

6. Alexandrov AV, Sloan MA, Wong LK, Douville C, Razumovsky AY, Koroshetz WJ, Kaps M, Tegeler CH. Practice standards for transcranial doppler ultrasound: part i-test performance. J Neuroimaging. 2007; 17:11-18. [PubMed: 17238867]

7. Stone CJ. Additive regression and other nonparametric models. Ann Stat. 1985; 13:689-705.

8. Sabayan B, Jansen S, Oleksik AM, van Osch MJ, van Buchem MA, van Vliet P, de Craen AJ, Westendorp RG. Cerebrovascular hemodynamics in alzheimer's disease and vascular dementia: a meta-analysis of transcranial doppler studies. Ageing Res Rev. 2011; 11:271-277. [PubMed: 22226802]

9. Balfors EM, Franklin KA. Impairment of cerebral perfusion during obstructive sleep apneas. Am J Respir Crit Care Med. 1994; 150:1587-1591. [PubMed: 7952619]

10. Lee KY, Sohn YH, Baik JS, Kim GW, Kim JS. Arterial pulsatility as an index of cerebral microangiopathy in diabetes. Stroke. 2000; 31:1111-1115. [PubMed: 10797173]

11. Xiong YY, Mok V, Wong A, Leung T, Chen XY, Chu WC, Soo Y, Fu JH, Ding D, Hong Z, Wong KS. Evaluation of age-related white matter changes using transcranial doppler ultrasonography. J Neuroimaging. 201110.1111/j.1552-6569.2011.00649.x

12. Lee KO, Lee KY, Lee SY, Ahn CW, Park JS. Lacunar infarction in type 2 diabetes is associated with an elevated intracranial arterial pulsatility index. Yonsei Med J. 2007; 48:802-806. [PubMed: 17963337]

13. Young T, Peppard PE, Gottlieb DJ. Epidemiology of obstructive sleep apnea: a population health perspective. Am J Respir Crit Care Med. 2002; 165:1217-1239. [PubMed: 11991871]

14. Wallace DM, Ramos AR, Rundek T. Sleep disorders and stroke. Int J Stroke. 201210.1111/j. 1747-4949.2011.00760

15. Buysse DJ, Hall ML, Strollo PJ, Kamarck TW, Owens J, Lee L, Reis SE, Matthews KA. Relationships between the Pittsburgh Sleep Quality Index (PSQI), Epworth Sleepiness Scale (ESS), and clinical/polysomnographic measures in a community sample. J Clin Sleep Med. 2008; 4:563-571. [PubMed: 19110886]

16. Jimenez-Correa U, Haro R, Gonzalez-Robles RO, Velazquez-Moctezuma J. How is the Epworth Sleepiness Scale related with subjective sleep quality and polysomnographic features in patients with sleep-disordered breathing? Sleep Breath. 2011; 15:513-518. [PubMed: 20559743]

17. Nieto FJ, Young TB, Lind BK, Shahar E, Samet JM, Redline S, D’Agostino RB, Newman AB, Lebowitz MD, Pickering TG. Association of sleep-disordered breathing, sleep apnea, and 
hypertension in a large community-based study. Sleep heart health study. JAMA. 2000; 283:18291836. [PubMed: 10770144]

18. Rasche K, Keller T, Tautz B, Hader C, Hergenc G, Antosiewicz J, Di Giulio C, Pokorski M. Obstructive sleep apnea and type 2 diabetes. Eur J Med Res. 2010; 15:152-156. [PubMed: 21147644]

19. Behrens S, Spengos K, Hennerici M. Acceleration of cerebral blood flow velocity in a patient with sleep apnea and intracranial arterial stenosis. Sleep Breath. 2002; 6:111-114. [PubMed: 12244490]

20. Virtanen J, Noponen T, Salmi T, Toppila J, Merilainen P. Impaired cerebral vasoreactivity may cause cerebral blood volume dip following obstructive sleep apnea termination. Sleep Breath. 2012; 16:309-312. [PubMed: 21553349]

21. Reichmuth KJ, Dopp JM, Barczi SR, Skatrud JB, Wojdyla P, Hayes D Jr, Morgan BJ. Impaired vascular regulation in patients with obstructive sleep apnea: effects of continuous positive airway pressure treatment. Am J Respir Crit Care Med. 2009; 180:1143-1150. [PubMed: 19745203]

22. Terborg C, Gora F, Weiller C, Rother J. Reduced vasomotor reactivity in cerebral microangiopathy: a study with near-infrared spectroscopy and transcranial doppler sonography. Stroke. 2000; 31:924-929. [PubMed: 10754000] 


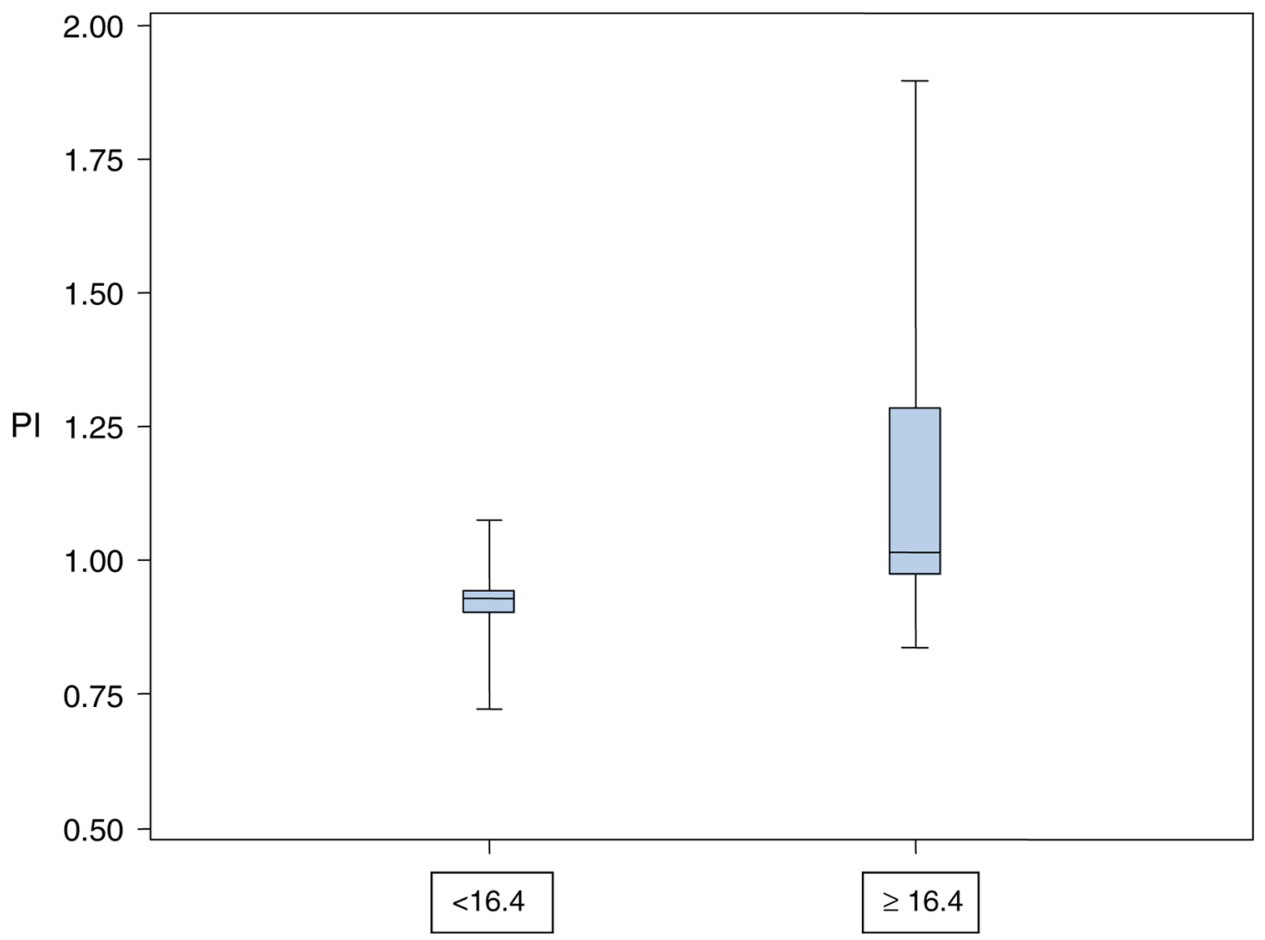

Fig. 1.

Pulsatility index by the median apnea-hypopnea index 
Table 1

Characteristics of the overall sample and groups divided by the median apnea-hypopnea index

\begin{tabular}{|c|c|c|c|}
\hline$N=19$ & All & $\mathrm{AHI}<16.4$ & $\mathrm{AHI} \geq 16.4$ \\
\hline Demographics & & $n=9$ & $n=10$ \\
\hline Age, years & $48.0(37-83)$ & $41.0(8.0)$ & $52.0(11.0)^{*}$ \\
\hline Men, $n(\%)$ & $10(52)$ & $3(33)$ & $7(70)$ \\
\hline $\mathrm{BMI}, \mathrm{kg} / \mathrm{m}^{2}$ & $29.9(25-40)$ & $32.1(5.6)$ & $28.1(5.9)$ \\
\hline Hypertension, $n(\%)$ & $9(33)$ & $3(33)$ & $6(60)$ \\
\hline Diabetes, $n(\%)$ & $2(11)$ & - & $2(20)$ \\
\hline Epworth sleepiness scale & $8(1-21)$ & $10(13)$ & $5(12)$ \\
\hline \multicolumn{4}{|c|}{ Daytime blood pressure measurements } \\
\hline Systolic, mm Hg & $122.0(103-154)$ & $120.0(14.0)$ & $128.5(25.0)$ \\
\hline Diastolic, $\mathrm{mm} \mathrm{Hg}$ & $79.0(65-97)$ & $79.0(7.0)$ & $79.5(12.0)$ \\
\hline Heart rate & $70.0(50-91)$ & $71.0(11.0)$ & $68.5(26.0)$ \\
\hline \multicolumn{4}{|l|}{ Transcranial Doppler, cm/s } \\
\hline Mean velocity & $47.8(32.4-68.6)$ & $50.1(12.3)$ & $45.6(11.6)$ \\
\hline Peak systolic & $80.6(51.1-141.0)$ & $81(17.3)$ & $75.0(19.5)$ \\
\hline End-diastolic & $30.5(19.1-47.7)$ & $34.6(9.0)$ & $28.6(7.4)^{*}$ \\
\hline Pulsatility & $0.97(0.72-1.89)$ & $0.93(0.04)$ & $1.01(0.31)^{*}$ \\
\hline \multicolumn{4}{|l|}{ Polysomnography } \\
\hline AHI & $16.4(0.2-69)$ & $9.4(5.7)$ & $26.8(28.1)^{*}$ \\
\hline O2 Nadir & $84(70-94)$ & $87.0(7.0)$ & $81.5(8.0)$ \\
\hline
\end{tabular}

Data presented as median (range or interquartile range)

* $p<0.05$ 